\title{
Temporal decorrelation of tropical dense forest at C-band: first insights from the TropiScat-2 experiment
}

\author{
S. El Idrissi Essebtey ${ }^{(1,2)}$, L. Villard ${ }^{(2)}$, P. Borderies ${ }^{(1)}$, T. Koleck ${ }^{(2)}$, J. P. Monvoisin ${ }^{(1)}$, B. Burban $^{(3)}$, T. Le Toan ${ }^{(2)}$ \\ (1) Office National d'Etudes et de Recherches Aérospatiales ONERA/DEMR, Université de Toulouse, 31055 Toulouse Cedex 4, France. \\ (2) Centre d'Etudes Spatiales de la BIOsphère (CESBIO), UMR UPS-CNRS-IRD-CNES, 31401 Toulouse Cedex France. \\ (3) Ecologie des Forêts de Guyane (EcoFog), UMR AgroParisTech-CIRAD-INRA-CNRS-Université de Guyane, Université des Antilles, \\ 97379 Kourou, Guyane Française.
}

\begin{abstract}
Following the past TropiScat and AfriScat tower-based scatterometer experiments conducted as part of the ESA BIOMASS mission preparation activities, a follow-on experiment referred to as TropiScat-2 has been undertaken in French Guiana since March 2018. Based on new capabilities including C-band acquisitions, this paper addresses the question of temporal decorrelation variability at several time scales using a period of 67 days dating back to early August 2018. Overall, C-band coherences have been found very dependent on the reference hour and higher than expected at night (15-min coherences above 0.75 and 1-hour coherences above $0.575 \%$ of the time). Supported by meteorological data from the flux-tower, clear evidence about diurnal convective effects is shown. For the first time the link with evapotranspiration is pointed out based on a drop of the 15-min coherences going from about 0.8 to 0.2 in less than 1 hour (around $7 \mathrm{h30}$ ) and before the rise of convective winds. Furthermore, the specific impacts of convective winds during the day have been demonstrated using very short timescales (with the spread of coherences at 1 sec from 0 to 0.9 ), whereas it is more difficult to separate these effects from those of water transfer for longer timescales. In addition to provide new insights for the understanding of microwave interactions with dense vegetation, these results should also contribute to widen the capabilities of future companion or high revisit space-borne missions at $\mathrm{C}$-band.
\end{abstract}

Index Terms-Temporal decorrelation; C-band; Time series; Tower-based scatterometer; Tropical dense forests; evapotranspiration; convective wind

\section{INTRODUCTION}

Time series measurements in remote sensing are often determinant to disentangle the possible ambiguities between the multiple causes behind signal variations, making revisit and continuity of time series highly important. Although limited by a rather small footprint (typically about $1 \mathrm{ha}$ ), tower based remote sensing instruments enable a top view of a given region of interest, and thereby allow to mimic observation geometry from space but with the core advantage of making possible a wide range of quasi-continuous and -simultaneous radar measurements, together with in situ auxiliary data. In the context of the ESA BIOMASS mission preparation activities, we can refer to the TropiScat [1] and AfriScat [2] experiments, respectively conducted in French Guiana (2011-2014) and Ghana (2015-2017). Both experiments were dedicated to tropical dense forests (primary targets of BIOMASS). The key results have been related to the signal variability at P and L-bands and particularly to the signal temporal decorrelation [3] which is a core variable for the design of mission revisit and acquisition time [4]. More recently, a similar experiment has been undertaken to address these questions in the case of a boreal forest [5], considering also C-band acquisitions in addition to the $\mathrm{P}$ and L-band ones. Indeed, several studies based on space-borne data (especially with ERS and ENVISAT missions) have shown the potential of C-band backscatter for boreal forests whether for seasonal monitoring and classification or even for AGB mapping $[6,7,8]$.

For tropical forests, the use of C-band to provide relevant information is less obvious. This situation can first be explained by the lack of data in comparison to areas at higher latitude where the revisits related to heliosynchronous orbits are more frequent. Moreover, canopies in the tropics are denser and vegetation cover fraction is higher resulting in a stronger attenuation and weaker image contrast. Finally, the phenology of the different tree species in tropical forests is much more diverse and poorly known [9]. Indeed, this phenology cannot be characterized by strong seasonal changes such as freeze/thaw events, leaf-on/leaf-off or even more progressive Leaf Area Index (LAI) changes as for temperate forests [10, 11].

Although challenging at seasonal scales, shorter revisit C-band observations might provide significant insights regarding the more predictable effects of convective winds or water content variations during the day, as pointed out before at $\mathrm{P}$ and $\mathrm{L}$ bands [12]. Concerning convective winds, strong effects can be indeed expected on temporal decorrelation, given that the induced displacements can easily reach half of the wavelength at C-band (about $3 \mathrm{~cm}$ ). On that matter, we can refer to several theoretical approaches either based on analytical models [12,13] or numerical ones [14] that relate temporal decorrelation to descriptive models of wind motions or sub-canopy wind fields, although the latter remains poorly known [15]. Likewise, important effects can be expected from the diurnal variations of water content and their associated dielectric values especially 
within the top canopy leaves which play an important role in both the C-band backscatter and the evapotranspiration (ET) phenomenon. In spite of many uncertainties about water content distribution within trees (from trunk to leaves), diurnal variations are related to ET which can be directly measured from the analysis of eddy covariance fluxes [16]. Alternatively, ET can be approximated by the Potential evapotranspiration (PET). The main assumption behind the use of PET lies in the availability of water resources. PET can be calculated through the widely used Penman formulation [17] based on temperature measurements, solar radiation and Vapour Pressure Deficit.

In view of these considerations, we propose in this paper to exploit the short revisit capabilities of TropiScat-2, in order to investigate the origins of temporal decorrelation at C-band. Next section is focused on the new facilities related to C-band acquisitions, while the statistical analysis of the coherences based on several methods to combine intervals from seconds and up to 30 days are explained in section III using also in situ wind and PET measurements. These results and associated questions are then discussed in section IV using supplementary data from a preliminary phase of the experiment. The conclusion section $\mathrm{V}$ includes further prospects related to the extent of these results.

\section{DATA ACQUISITION AND PROCESSING}

\section{A. New features of the experiment to include C-band acquisitions}

As introduced above, a similar P- and L-bands antenna array as for the previous TropiScat and AfriScat campaigns has been installed at the top of the Guyaflux tower (about 55m high) in French Guiana [18]. To extend the measurements to Cband, a new Vector Network Analyzer (VNA, ref ENA-E5063A detailed on http://www.keysight.com) has been installed, covering a wide range from $5 \mathrm{kHz}$ to $6.5 \mathrm{GHz}$. For the period hereinafter considered in this study (67 days from August $1^{\text {st }}$ to October $\left.6^{\text {th }}, 2018\right)$, six antennas dedicated to C-band acquisitions have been set up at the VV polarization and equally split on two sides of the tower (west and northeast). This choice was made to ensure the reproducibility of our results, given the smaller vegetation volumes interacting at C-band compared to lower frequencies. The main features of these antennas are provided in $T a b$. 1. In the following, the west side pairs of transmit $(\mathrm{T})$ and receive $(\mathrm{R})$ antennas are noted R1-T1 and R2-T1, while the northeast ones are noted R3-T2 and R4-T2. For each side, both pairs have been respectively separated by a vertical baseline of $50 \mathrm{~cm}$ and $80 \mathrm{~cm}$ in view of further interferometric studies.

\begin{tabular}{|c|c|c|c|c|}
\hline $\begin{array}{c}\mathbf{f}_{\mathbf{0}} \\
(\mathbf{G H z})\end{array}$ & $\begin{array}{c}\text { Gain } \\
(\mathbf{d B})\end{array}$ & $\begin{array}{c}\text { Antenna } \\
\text { Factor }(\mathbf{d B} / \mathbf{m})\end{array}$ & $\begin{array}{c}\text { Cross-Pol } \\
\text { Isolation }(\mathbf{d B})\end{array}$ & $\begin{array}{c}\text { E-\&H-plane; } \\
\boldsymbol{\theta} \_3 d B\left({ }^{\circ}\right)\end{array}$ \\
\hline 5.0 & 10.51 & 33.68 & -34 & $56.77 \& 55.16$ \\
\hline 5.5 & 10.95 & 34.07 & -40 & $54.45 \& 49.93$ \\
\hline 6.0 & 11.63 & 34.14 & -44 & $50.57 \& 46.56$ \\
\hline
\end{tabular}

Tab. 1: Features of the horn antennas used for the C-band acquisitions (from 5.2 to $6 \mathrm{GHz}$ within the total bandwidth $4.90-7.05 \mathrm{GHz}$ ). The indicated frequencies and associated performances are those provided by our supplier (for more information, see model 'LB-159-10' on http://www.ainfoinc.com).

Each receive-transmit pair is connected to the dual port VNA through RF switches that sequentially route the signal. The acquisition is made in the frequency band $5.2-5.6 \mathrm{GHz}$ with 1601 samples, and is repeated 3 times (sequences $\mathrm{A}, \mathrm{B}, \mathrm{C}$ ) in order to increase the number of looks ( $c f$. §I. B.). The full acquisition cycles are performed every 15 minutes, except during the preliminary campaign (in March 2018) when 1-second and 1-minute tests have been performed (providing the complementary results in $\S I V$.). The two periods considered in this study were defined by the planning of on-site campaigns. No particular event regarding meteorological conditions has been reported locally and the cumulative rainfall amount of 156 $\mathrm{mm}$ during the 67-day period can be considered as ordinary in this region and for that period of the year (belonging to the dry season).

\section{B. Coherence computation}

Considering that data acquisition from the VNA is in the frequency domain, the processing consists first in an inverse fast Fourier transform to convert the measurements into the time domain. The resulting range impulse responses can then be used to compute the temporal coherence according to the following formula:

$$
\gamma=\frac{\sum_{k=1}^{K} \sum_{s e q}^{\{A, B, C\}} \sum_{i \min }^{i \max } S_{\text {seq,t1 }}^{k}(i) \cdot S_{\text {seq,t2 }}^{k}(i)^{*}}{\sqrt{\sum_{k=1}^{K} \sum_{\text {seq }}^{\{A, B, C\}} \sum_{i \min }^{i m a x}\left|S_{\text {seq,t1 }}^{k}(i)\right|^{2} \sum_{k=1}^{K} \sum_{\text {seq }}^{\{A, B, C\}} \sum_{i \min }^{i \max }\left|S_{\text {seq, } t 2}^{k}(i)\right|^{2}}}
$$

with ' $i$ ' being the range index between $i_{\min }$ and $i_{\max }$, and $S_{\text {seq,t1 }}$ and $S_{\text {seq,t2 }}$ being the complex responses for acquisition times $t_{1}$ and $t_{2}$. The index 'seq' is for the consecutive sequences $\mathrm{A}, \mathrm{B}, \mathrm{C}$ within a same acquisition cycle and ' $\mathrm{k}$ ' to cover the aforementioned antenna pairs. Considering a slant range interval of $15 \mathrm{~m}$ (40 pixels), the 3 sequences $\mathrm{A}, \mathrm{B}, \mathrm{C}$ and the 2 antenna pairs on each side of the tower, we come up with 240 non-independent looks. This number can be indeed compared to the Equivalent Number of Looks (ENL). In our case, the ENL can be estimated from the square ratio between the mean and the standard deviation [19] computed from the set of the $\left(i_{\max }-i_{\min }\right)$ spatial pixels within 1-D intensity images. Applying 
this method to 1-D multi-look images resulting from the 3 sequences and the two antennas pairs, we come up with variable values of ENL higher than $8075 \%$ of the time at night, and significantly higher during the day due to the decorrelation between the 3 sequences. Without anticipating the data analysis coming in the next sections, we can stress according to [20] that even a minimum ENL of 80 ensures a negligible bias for coherences above 0.2, and a bias below 0.1 for coherences below 0.2 .

For completeness, it can be also highlighted that formula (1) was used to process all results belonging to the main period (67 days from August $1^{\text {st }}$ ) whereas the complementary results provided in the discussion section ( $\$ I V$.) were limited to a single pair and a single sequence but with larger bandwidth $(1 \mathrm{GHz})$.

\section{ANALYSIS OF TEMPORAL DECORRELATION AT VARIOUS Time SCALES}

\section{A. Overview of the whole set of temporal coherence}

To visualize the overall variations of the temporal coherence, all possible combinations of time pairs $\left(\mathrm{t}_{1}, \mathrm{t}_{2}\right)$ can be gathered into a matrix representation. Columns and lines of such matrix correspond to all acquisition times from the beginning to the end of a selected period. Such matrix representation of temporal coherences is shown in Fig. 1, restricted to the period from August $20^{\text {th }}$ to $26^{\text {th }}$ for a better visualization. The upper and lower triangular parts represent results from the two antenna pairs R3-T2 and R4-T2, respectively (hence the slight dissymmetry). The diagonal elements of the matrix correspond to the zero temporal baseline. Thus, the corresponding coherence values are exactly equal to 1 as shown by a thin red line. As expected, the highest values are spread in the surrounding of the main diagonal during night-time. More interestingly, a periodicity of bright blocks can be pointed out when moving away from the diagonal (i.e. when the temporal baseline increases), revealing the effects of day and night conditions. Indeed, coherences during the day are mostly very low (below 0.2) while values often above 0.5 along the sub-diagonals can be noticed at nights. Given these first qualitative observations, specific temporal baselines and reference times can be chosen in order to quantify the decorrelation, as proposed in the following subsections.

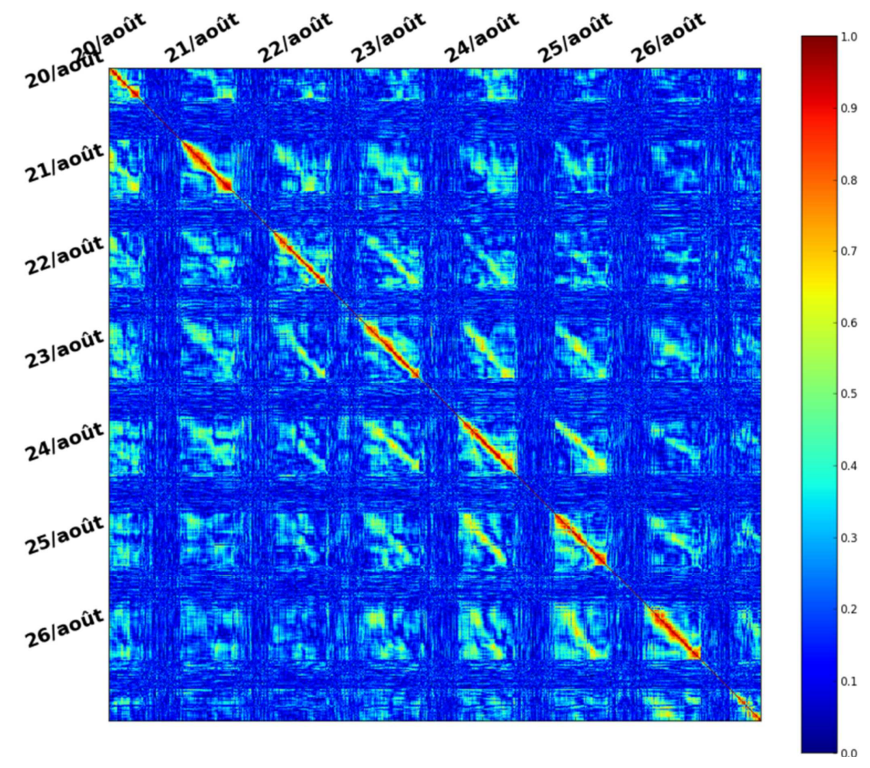

Fig. 1: Overview of the evolution of temporal decorrelation during one week (from August $20^{\text {th }}$ at midnight to $26^{\text {th }}$ at 11:45pm) for the West side of the fluxtower. The 2 antenna pairs are represented on each side of the diagonal.

\section{B. Daily variations of 15-minute coherences}

To better understand the daily variations of temporal coherence and to underline the aforementioned contrasts between day and night-time coherences, 15-minute coherences have been computed among all possible pairs within the whole period (01/08 to 06/10) and have been further reduced to a 24-hour scale to provide the results shown in Fig. 2, top graph. Likewise, PET and wind speed measurements (estimated from the Guyaflux data [18]) have been reduced for the whole period to 24 hours (Fig. 2, middle and bottom graphs). Given the daily evolution of the 15-minute coherences (top graph), three periods can be highlighted: the first one (midnight-7am) is characterized by a low temporal decorrelation with coherence values mostly higher than 0.8 (50\% of the time). Considering the fairly constant median during this period, it is very likely that the 0.2 decorrelation is mainly due to the residual wind induced motions. Indeed, assuming a decreasing water redistribution 
process due to ET from the previous day, we would expect, in the case of water content effect, a variation or a discontinuity of the coherences instead of the observed plateau. We can also stress that this hypothesis holds only for the top part of the forest which is involved in C-band interactions. The second period $(7 \mathrm{am}-7 \mathrm{pm})$ is then characterized by a sudden drop (around 7:15am) which coincides with the PET increase while the wind speed reaches a minimum (see the red strip in Fig. 2), suggesting that ET by itself can explain the coherence drop. The plateau of low coherences (below 0.2) continues until the slowdown of convective effects (around 7pm) before a more progressive and scattered coherence rise than the 7:15am drop. This dissymmetry is likely due to stronger wind speeds (mostly above $2 \mathrm{~m} / \mathrm{s}$ between $6 \mathrm{pm}$ and $9 \mathrm{pm}$ ) than during the drop period and also to possible lag of water redistribution within the trees during the evening. The analysis of Fig. 2 is further discussed in section $I V$.

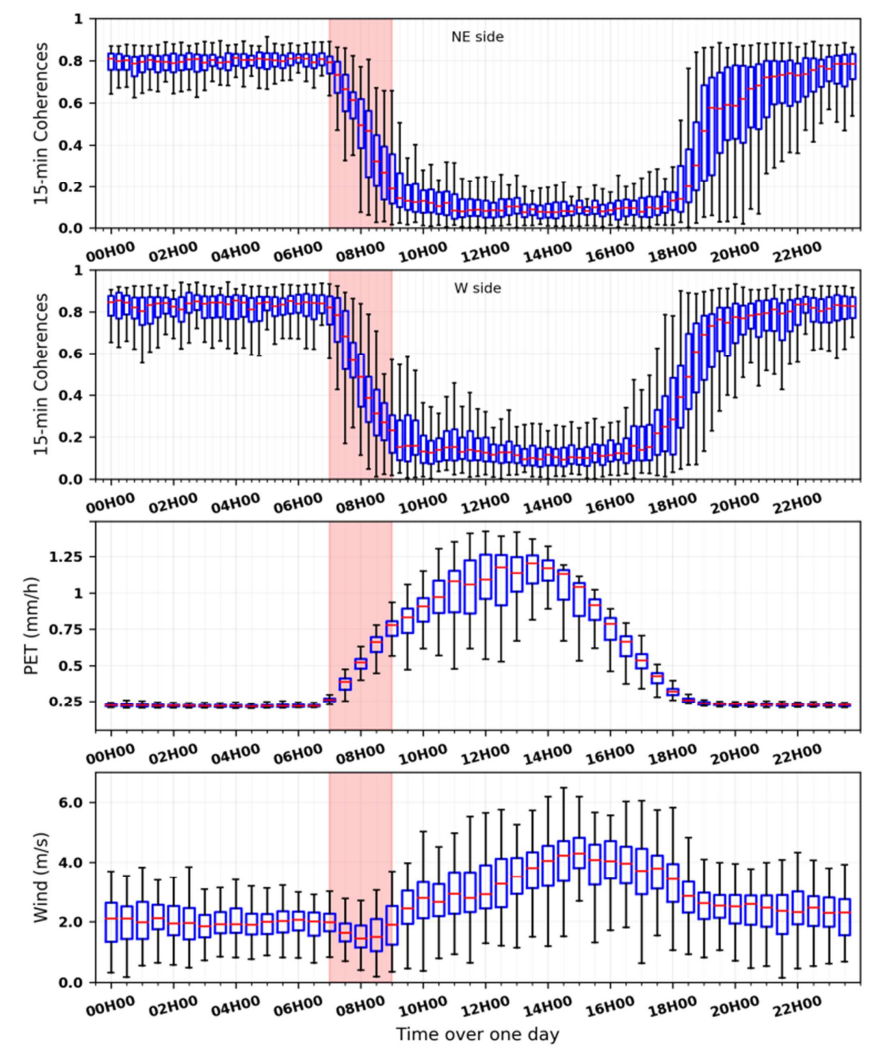

Fig. 2: From top to bottom, whisker-boxes (min, $1^{\text {st }}$ quartile, mean, $3^{\text {rd }}$ quartile and max) of 15-min coherences on the NE then the W sides, averaged PET and wind speed. For each sub-graph, results have been obtained using the whole period (August $1^{\text {st }}$ to October $6^{\text {th }}, 2018$ ), reduced to a 24-hour scale.

\section{Coherence evolution from 1 to 30 days for selected hours}

To investigate the possible high coherences between several days, specific reference hours have been selected (midnight, 6am and $6 \mathrm{pm}$ ) to compute all combinations of coherences for a temporal baseline ranging from 1 to 30 days. The associated results are shown in Fig. 3. They confirm the more favorable conditions at night or early morning, showing a slightly exponential decay (top and middle graphs) from truly interesting values up to 0.6 (and above $0.425 \%$ of the time) to low values (around 0.2 ) after 4 days. On the contrary, the $6 \mathrm{pm}$ reference (bottom graph) is quasi time invariant except for the 1 day coherence reaching 0.4 as a maximum.

\section{Coherence evolution from 15 minutes to 5 hours during day, evening \& night-time}

To complete the previous findings, smaller ranges of temporal baseline can be analysed using 15-minute to 4- or 5-hour intervals for the three aforementioned selected periods (resulting from the analysis of Fig. 2) and are respectively shown in Fig. 4. As expected from the previous analysis, great differences between the evolution of temporal coherences during the day and those during evening and night-time can be noticed. Indeed, the median of the coherences computed during the day is already below 0.2 after $15 \mathrm{~min}$ and only the maxima show an exponential like decay. On the contrary, coherences during night-time (midnight to $7 \mathrm{am}$ ) or during the evening ( $7 \mathrm{pm}$ to midnight) are mostly above 0.2 even after 4 hours. The main 
difference between both periods lies in the dispersion of the coherences at 15, 30 and 45 minutes, with the minima being significantly higher for the night (above 0.4 vs zero) as well as the first quartiles (above 0.6 vs 0.4 ).

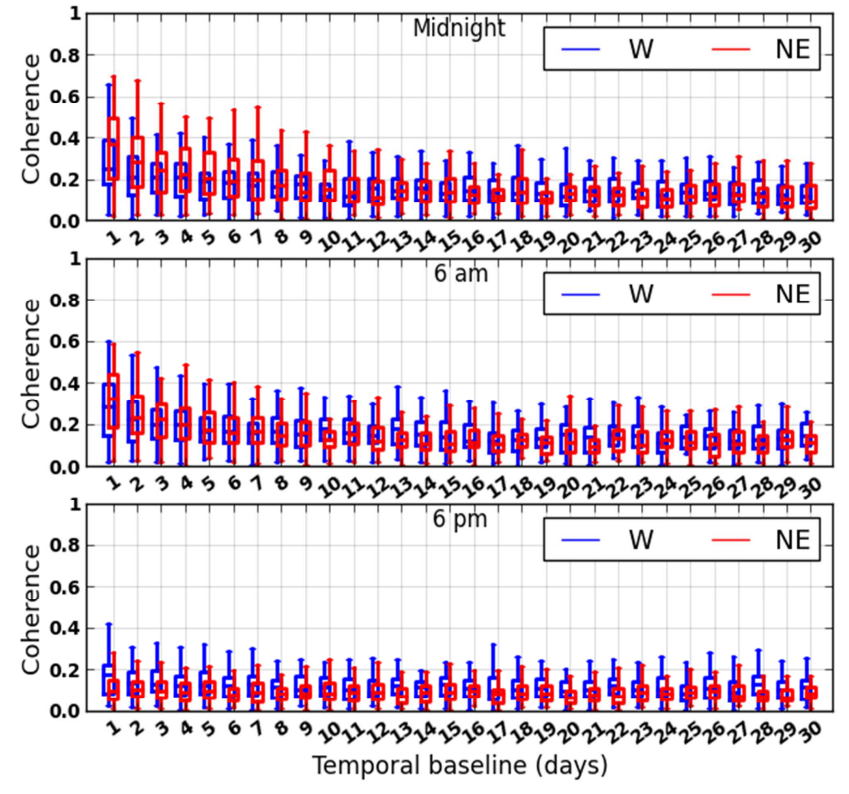

Fig. 3: From top to bottom, statistical (whisker-boxes) evolution of temporal decorrelation at fixed time: "midnight", "6am" and "6pm" versus temporal baseline for both sides of the tower (represented with a slight shift for clarity).
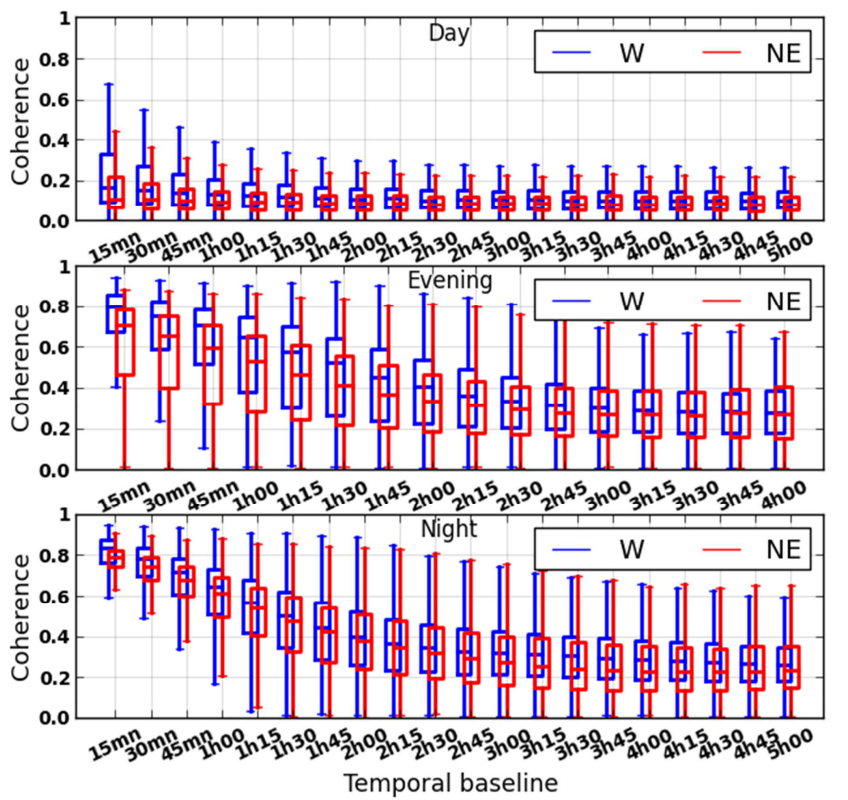

Fig. 4: From top to bottom, statistical (whisker-boxes) evolution of temporal decorrelation for three periods: "day", "evening" and "night", versus temporal baseline, for both sides of the tower (represented with a slight shift for clarity as in Fig. 3).

\section{DisCUSSION ENLIGHTENED WITH COMPLEMENTARY RESULTS}

Considering the previous results related to the convective effects on the 15-minute coherences (cf. Fig. 2), we saw the difficulty to separate both effects in the slot between $9 \mathrm{am}$ and $6 \mathrm{pm}$. Indeed, we might explain the slight decrease of the 15minute coherences after $1 \mathrm{pm}$ by an increase of convective wind while PET remains constant (near its maximum) or decreases, assuming in this case less effects on vegetation water content and associated dielectric changes (of the vegetation scatterer) which directly impact the backscatter. However, this assumption is not yet verified since evapotranspiration only reflects how conditions are favorable for water circulation and dielectric changes but it is not a direct measurement of vegetation water content. Future longer time series might help us isolate both effects with possible occurrence of unusual low/high wind conditions during the day/night-time.

As an alternative, the possibility to estimate coherences at shorter time scales can help us dissociate wind and evapotranspiration effects, assuming that the latter does not vary so quickly. For that purpose, previous measurements can be exploited from a preliminary campaign (March 2018) which aimed at the design of the instrument during preparation phases. In fact, higher sampling rates have been performed during a short period of time since they could not be afforded during the long-term nominal mode (regarding data storage and signal amplification to compensate shorter integration time). These experiments enabled to compute temporal coherences among measurements acquired every second during the day (from 9:30am to $12: 30 \mathrm{pm}$ ) and every minute during night-time (from midnight to 6am) as shown in the two graphs in Fig. 5 . Interestingly, decorrelation at 1 or 2 seconds can be really high (maxima above 0.8 ) and are also characterized by a high dispersion (first and third quartiles around 0.2 and 0.6 respectively) which is very likely due to the quasi-random displacements caused by wind (assuming again a constant evapotranspiration at one or several seconds). During this slot, it should also be noted that wind was particularly strong with all recorded values above $3.65 \mathrm{~m} / \mathrm{s}$. Beyond this important spread of the coherences, these results also indicate that tandem missions cannot exceed a shift of one second (i.e baseline of about $7.5 \mathrm{~km}$ ) or should head for alternative configurations with twin satellites flying in close formation (as proposed with Sentinel1 in [21]). During night-time (bottom graph), 1-min coherences are much higher than during the day (first quartile close to 0.8 ) and coherences for longer intervals are consistent with those found using the main period (cf. bottom graph in Fig. 4). 


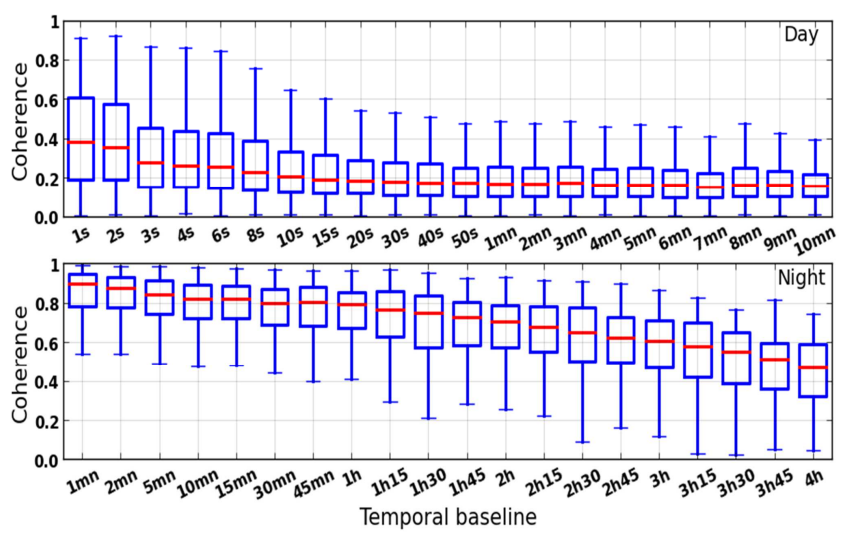

Fig. 5: Statistical evolution of temporal decorrelation at day (top) and night (bottom) versus temporal baseline.

\section{CONCLUSION AND FurTher PROSPECTS}

Supported by a wide-range time intervals (from seconds to days), higher than expected C-band coherences have been found in the case of tropical forest. Their evolution has been not only explained by convective motions but also by evapotranspiration. Indeed, while most previous studies highlighted the effects of wind motions, this paper demonstrates that evapotranspiration also matters and is even the main driver of the coherence drop in the early morning when convective winds are minimum. After this minimum and as long as convection remains high, the combined effects of wind and evapotranspiration remain difficult to disentangle. However, the high spread of the 1-sec coherences (from 0 to 0.9 ), observed with a dedicated mode, indicates that wind motions are very likely to be the main source of decorrelation since evapotranspiration cannot change so fast. As expected, longer intervals during the day lead to lower coherences (mostly around 0.2 ). The greatest coherences have been obtained at night with 15 -min coherences around 0.8 and more surprisingly above 0.2 after one day (reaching $0.425 \%$ of the time). Considering that coherences above 0.2 can be exploited in different applications based on coherence magnitude and phase, these observations open many opportunities for innovative configurations of future high revisit geostationary, tandem or formation-flying missions.

Besides, having in mind the current and upcoming TropiScat-2 acquisitions, further work will be specifically dedicated to the analysis of the interferometric phase time series, benefiting also from the recently available polarimetric capabilities. Longer time series will also provide a wider range of environmental conditions to better disentangle wind and evapotranspiration effects (especially with possible LAI variations and the analysis of dry and wet seasons), in addition to the more general objectives related to potential synergies between the backscatter (intensity and phase) at P, L and C-bands. On that matter, the highlighted importance of evapotranspiration is very encouraging since it may relate $\mathrm{P}, \mathrm{L}$ and $\mathrm{C}$-band data thanks to the continuum of water content within the trees.

\section{ACKNOWLEDGMENTS}

This work has been supported by the French national space agency (CNES) through the research contributions program TOSCA. The authors are very thankful to all the people at EcoFog (Laboratoire d'Ecologie des Forêts de Guyane, Kourou) contributing to the success of the Paracou research station. The authors are also grateful to the reviewers for their relevant comments and overall interest.

\section{REFERENCES}

[1] A. Hamadi, P. Borderies, C. Albinet, et al. Temporal coherence of tropical forests at P-band: Dry and rainy seasons. IEEE Geoscience and Remote Sensing Letters, 2015, vol. 12, no 3, p. 557-561.

[2] AfriScat Final Report, ESA CONTRACT N $4000111758 / 14 / \mathrm{NL} / \mathrm{FF} / \mathrm{GP}$

[3] A. Hamadi, L. Villard, P. Borderies, et al. Comparative Analysis of Temporal Decorrelation at P-Band and Low L-Band Frequencies Using a TowerBased Scatterometer Over a Tropical Forest. IEEE Geoscience and Remote Sensing Letters, 2017, vol. 14, no 11, p. 1918-1922.

[4] T. Le Toan, S. Quegan, et al. The BIOMASS mission: Mapping global forest biomass to better understand the terrestrial carbon cycle. Remote sensing of environment, 2011, vol. 115, no 11, p. 2850-2860.

[5] L.M.H. Ulander, A. R. Monteith, et al. Multiport Vector Network Analyzer Radar for Tomographic Forest Scattering Measurements. IEEE Geoscience and Remote Sensing Letters, 2018, no 99, p. 1-5.

[6] J.I.H. Askne, P.B.G. Dammert, L.M.H. Ulander, et al. C-band repeat-pass interferometric SAR observations of the forest. IEEE Transactions on Geoscience and Remote Sensing, 1997, vol. 35, no 1, p. 25-35.

[7] W. Wagner, J. Vietmeier, et C. Schmullius. Information content of ERS SAR interferometric products for forest classification in SIBERIA: a case study over the Bolshemurtinskii forest enterprise. In: Geoscience and Remote Sensing Symposium 2000. p. 444-446. 
[8] M. Santoro, J. Askne, G. Smith, et al. Boreal forest monitoring with ERS coherence. In: Proc. ERS-Envisat Symposium. 2000. p. 16-20.

[9] J. Wu, L.P. Albert, A.P. Lopes, et al. Leaf development and demography explain photosynthetic seasonality in Amazon evergreen forests. Science, 2016, vol. 351, no 6276, p. 972-976.

[10] C. Proisy, E. Mougin, A. Lopes, et al. Usefulness of ERS coherence time series for monitoring the vegetation cycle of a mixed deciduous forest. Proc. ERSENVISAT symposium, Gothenburg. 2000. p. 16-20.

[11] P-L. Frison, B. Fruneau, S. Kmiha, et al. Potential of Sentinel-1 Data for Monitoring Temperate Mixed Forest Phenology. Remote Sensing, 2018, vol. 10 , no 12, p. 2049.

[12] J.IH. Askne, P.B.G. Dammert, L.M.H Ulander, et al. C-band repeat-pass interferometric SAR observations of the forest. IEEE Transactions on Geoscience and Remote Sensing, 1997, vol. 35, no 1, p. 25-35.

[13] M. Lavalle, M. Simard, and S. Hensley. A temporal decorrelation model for polarimetric radar interferometers. IEEE Transactions on Geoscience and Remote Sensing, 2012, vol. 50, no 7, p. 2880-2888.

[14] L. Villard, T. Le Toan, D.H.T. Minh, et al. Forest biomass from radar remote sensing. In: Land Surface Remote Sensing in Agriculture and Forest. Elsevier, 2016. p. 363-425.

[15] M.R. Raupach, J.J. Finnigan, and Y. Brunet. Coherent eddies and turbulence in vegetation canopies: the mixing-layer analogy. In: Boundary-Layer Meteorology 25th Anniversary Volume, 1970-1995. Springer, Dordrecht, 1996. p. 351-382.

[16] M. Aguilos, B. Hérault, B. Burban, et al. What drives long-term variations in carbon flux and balance in a tropical rainforest in French Guiana? Agricultural and forest meteorology, 2018, vol. 253, p.114-123.

[17] A. Granier, R. Huc, and S.T. Barigah. Transpiration of natural rain forest and its dependence on climatic factors. Agricultural and forest meteorology, 1996, vol. 78, no 1-2, p. 19-29.

[18] Guyaflux description: https://paracou.cirad.fr/website

[19] S. Quegan and J.J. Yu. Filtering of multichannel SAR images. IEEE Transactions on Geoscience and Remote Sensing, 2001, vol. 39, no 11, p. 23732379.

[20] R. Touzi, A. Lopes, J. Bruniquel et al. Coherence estimation for SAR imagery. IEEE Transactions on Geoscience and Remote Sensing, 1999, vol. 37, no 1 , p. 135-149.

[21] P. López-Dekker, H. Rot, S. Solberg, et al. Companion SAR constellations for single-pass interferometric applications: The SESAME mission. In: Geoscience and Remote Sensing Symposium (IGARSS), 2017 IEEE International. IEEE, 2017. p. 119-122. 